\title{
A Stability Analysis of Fifth-Order Water Wave Models
}

\author{
Steven Paul Levandosky \\ Department of Mathematics \\ Stanford University
}

\begin{abstract}
We study the stability of traveling wave solutions to a fifth-order water wave model. By solving a constrained minimization problem we show that "ground state" traveling wave solutions exist. Their stability is shown to be determined by the convexity or concavity of a function $d(c)$ of the wave speed $c$. The analysis makes frequent use of the variational properties of the traveling waves.
\end{abstract}

\section{Introduction}

We study the stability properties of traveling wave solutions to a generalized fifth-order Korteweg-deVries equation of the form

$$
u_{t}+u_{x x x x x}+b u_{x x x}=\left(f\left(u, u_{x}, u_{x x}\right)\right)_{x}
$$

where we assume the nonlinear term has the variational structure

$$
f(q, r, s)=F_{q}(q, r)-r F_{q r}(q, r)-s F_{r r}(q, r)
$$

for some $F(q, r) \in C^{3}\left(\mathbb{R}^{2}\right)$ which is homogeneous of degree $p+1$ for some $p>1$. That is, we assume

$$
F(\lambda q, \lambda r)=\lambda^{p+1} F(q, r)
$$

for all $\lambda \geq 0$ and $(q, r) \in \mathbb{R}^{2}$.

Such equations arise as long-wave approximations to the water-wave equations. On the one hand, they have been derived as second order asymptotic expansions for unidirectional wave propagation, the first order expansions being of course the Korteweg-deVries equation

$$
u_{t}+b u_{x x x}+u u_{x}=0 .
$$

In this way Olver [23] derived equation (1.1), with a nonlinear term of the form $F\left(u, u_{x}\right)=$ $-u u_{x}^{2}$ (for a specific choice of parameters). The same equation was proposed by Benney [5] as a model for the interaction of short and long waves. Later, both Zufiria [31] and Hunter \& Scheurle [13] derived (1.1) with $F\left(u, u_{x}\right)=-u^{3}$, and considered it as a model for water waves 
with Bond number $\tau$ near $1 / 3$, the critical value at which the KdV equation ceases to be an effective model. In both (1.1) and (1.4) the coefficient $b$ of the third order dispersive term is proportional to $\tau-1 / 3$. On the other hand, higher-order model equations have been derived by considering perturbations of the Hamiltonian structure of the full water-wave problem. In [23] Olver obtained (1.1) with the (inhomogeneous) nonlinear term $F\left(u, u_{x}\right)=u^{4}+u u_{x}^{2}$. More recently Craig \& Groves [11] observed that the Hamiltonian structure of the waterwave problem may be formulated using the Dirichlet-Neumann operator. By considering a Taylor expansion of this operator they arrived at (1.1) with $F\left(u, u_{x}\right)=u u_{x}^{2}-u^{3}$. For a more detailed discussion of the history of higher-order water wave models equations we refer the reader to $[17,24]$ and the references therein. We remark that, due to the homogeneity assumption on $F$, the class of equations considered here does not include equations in the so-called KdV heirarchy discovered by Lax [18].

By a traveling wave we mean a solution of (1.1) of the form $u(x, t)=\varphi(x+c t)$, where $c$ represents the speed of the wave. Inserting this into (1.1) and integrating once, we see that $\varphi$ must satisfy

$$
\varphi_{x x x x}+b \varphi_{x x}+c \varphi=f\left(\varphi, \varphi_{x}, \varphi_{x x}\right)
$$

This equation also appears as the solitary wave equation for models of both a buckling strut $[3,25]$ and a suspension bridge [22]. A higher dimensional analog has also been considered [19]. Furthermore, we mention that equation (1.5) (with $f\left(\varphi, \varphi_{x}, \varphi_{x x}\right)=\varphi^{2}$ ) has been derived directly as a model for solitary water-waves by means of a center-manifold reduction of the full water-wave equations $[2,8]$. In this paper, however, we consider only the stability properties of traveling waves with respect to the evolution equation (1.1).

The existence of traveling wave solutions of (1.1) has been has been considered by many authors. Using techniques identical to those presented here, Weinstein [30] proved existence in the case $b<0$ and $F\left(u, u_{x}\right)=|u|^{p+1}$. For $F\left(u, u_{x}\right)=-u^{3}$ existence was established in [31] and [13]. Kichenassamy and Olver [17] proved criteria for the existence of $\operatorname{sech}^{2}$ type solitary waves, and showed that such solutions exist only if $f\left(u, u_{x}, u_{x x}\right)$ is a cubic polynomial. Kichenassamy [16] proved the existence of traveling wave solutions of (1.1) for the nonlinearity of the form $F\left(u, u_{x}\right)=-u u_{x}^{2}+u^{3}+u^{4}$. His method of proof used a variational argument similar to the one presented here. The results however differ in two respects: first, the nonlinearity he considered was inhomogeneous while those considered here are all homogeneous, and second, the speed of the solitary waves found was given by a Lagrange multiplier, while here the speed is specified as a parameter in the functionals used in the minimization. Recently Champneys and Groves [10] considered traveling waves of (1.1) with a nonlinearity of the form $F\left(u, u_{x}\right)=u u_{x}^{2}-u^{3}$ derived in [10]. They arrived at a global (in the paramater $b$ and wave speed $c$ ) bifurcation picture which describes the regions in which traveling waves exist, as well as the types of traveling waves (e.g. elevation, depression, multi-modal) which exist in each region. The most notable feature of the traveling waves treated here is their lack of positivity. The values of $b$ and $c$ we consider here are such that the linearization of (1.5) about zero has four complex eigenvalues. Thus the solitary waves have exponentially decaying oscillatory tails, and are therefore non-positive. In the case $F\left(u, u_{x}\right)=u u_{x}^{2}-u^{3}$ numerical evidence shows these traveling waves to be waves of depression [10].

Stability results for solitary waves of the Korteweg-deVries equation go back to the works 
of Benjamin [4] and Bona [6]. They showed that solitary wave solutions are stable in the space $H^{1}(\mathbb{R})$ associated with the conserved quantities of (1.4). Later, Bona, Souganidis and Strauss [7] and Souganidis and Strauss [28] obtained stability and instability results for certain generalizations of these equations by making use of the Hamiltonian formulation of these problems to apply the theory of Grillakis, Shatah and Strauss [12]. By a different method, Weinstein $[29,30]$ proved stability of solitary waves for a class of generalized longwave equations. The results of this paper are in the same spirit as those above, in that we consider the nonlinear stability (see Definition 1.3) of traveling wave solutions of (1.1) in the space $H^{2}(\mathbb{R})$ associated with its conserved quantities. We show that there exists a function $d(c)$ of the wave speed $c$ such that traveling waves with speed $c$ are stable if and only if $d$ is convex at $c$. See Theorems 4.1, 5.3 and Corollary 5.14.

Much of the stability analysis is based on techniques developed in the works cited above. The most noteworthy difference concerns the assumptions made in [7, 28, 30] about the traveling wave solutions $\varphi$. First, it was assumed that the traveling waves are positive which, as mentioned above, is not the case here. Second, it was assumed that the operator $H_{c}$ (see Definition 5.1) obtained by linearizing the solitary wave equation about the traveling wave $\varphi$ has exactly one negative eigenvalue and that zero is a simple eigenvalue with eigenfunction $\varphi_{x}$. This last assumption is particularly difficult to verify in practice $[1,29,12]$. To avoid these difficulties, we use the variational characterization of the traveling waves. The stability proof uses a compactness argument due to Cazenave \& Lions [9] and Shatah [26]. The proof of instability is an application of the theory of Grillakis, Shatah and Strauss [12], modified as in [7] to fit the present problem. The key observation in both cases is that the invariants of (1.1) and the functionals by which the traveling waves are obtained are related by equation (3.1). This ideas was also used by the author in the stability analysis of a fourth-order wave equation [19] and by Liu [21], who studied the stability of the KP equation. Shatah \& Strauss [27] used a similar technique to prove the instability of solitary waves for the generalized Klein-Gordon equation.

The existence of traveling waves is considered in Section 2. We construct solutions of (1.5) by solving a constrained minimization problem in $H^{2}(\mathbb{R})$. The concentration-compactness lemma of Lions [20] is used to show that all minimizing sequences for this problem are relatively compact in $H^{2}(\mathbb{R})$ up to a sequence of spatial translations. This fact, in addition to establishing existence of traveling waves, becomes a central feature in both the regularity of $d(c)$ and the proof of the stability theorem. We show that the traveling waves thus obtained are classical solutions of (1.5). In Section 3 we define the function $d(c)$ of the wave speed $c$ by which the stability of the traveling waves is determined. We establish some regularity properties of $d(c)$ and derive an explicit formula for the case when $b=0$ and $F(q, r)$ is jointly homogeneous in $q$ and $r$. Section 4 contains the proof of the main stability theorem and uses a compactness argument due to Cazenave \& Lions [9] and Shatah [26]. The instability theorem is proved in Section 5. Applying the theory of Grillakis, Shatah and Strauss [12] (see also [7] and [28]) we construct a Lyapunov functional. The construction of the function $\lambda$ in Lemmas 5.8 and 5.9 is where equation (3.1) is used in place of spectral information. 
The functionals

$$
\begin{aligned}
& E(u)=\int_{-\infty}^{\infty} \frac{1}{2}\left|u_{x x}\right|^{2}-\frac{b}{2}\left|u_{x}\right|^{2}-F\left(u, u_{x}\right) d x \\
& Q(u)=\frac{1}{2} \int_{-\infty}^{\infty}|u|^{2} d x
\end{aligned}
$$

are in $C^{2}\left(H^{2}(\mathbb{R}), \mathbb{R}\right)$ and are (formally) conserved quantities of (1.1). In terms of $E$ the evolution equation (1.1) may be written

$$
u_{t}=J E^{\prime}(u)
$$

where $J=-\partial_{x}$, while the traveling wave equation (1.5) takes the form

$$
E^{\prime}(\varphi)+c Q^{\prime}(\varphi)=0
$$

We make the following assumption concerning the well-posedness of $(1.1)$ in the space $H^{2}(\mathbb{R})$.

Assumption 1.1 Given initial data $g \in H^{2}(\mathbb{R})$ there exists some $T>0$ which depends only on $\|g\|_{H^{2}(\mathbb{R})}$ and a unique solution $u \in C\left([0, T), H^{2}(\mathbb{R})\right)$ of $(1.1)$ with $u(0)=g$, which satisfies $E(u(t))=E(g), Q(u(t))=Q(g)$ for $t \in[0, T)$.

Remark 1.2 When $F\left(u, u_{x}\right)$ depends only on $u$, Assumption 1.1 follows by applying the results of Kato [14],[15]. For certain more general nonlinearities Ponce [24] has shown wellposedness in $H^{s}(\mathbb{R})$ for $s \geq 4$.

We will use the following definition of stability throughout.

Definition 1.3 We say a set $S \subset H^{2}(\mathbb{R})$ is stable with respect to (1.1) if given $\varepsilon>0$ there exists some $\delta>0$ such that if $g$ satisfies $\inf _{\psi \in S}\|g-\psi\|_{H^{2}(\mathbb{R})}<\delta$ then the solution $u(t)$ of (1.1) with initial data $u(0)=g$ exists for all $t>0$ and satisfies $\sup _{t \in[0, \infty)} \inf _{\psi \in S}\|u(t)-\psi\|_{H^{2}(\mathbb{R})}<\varepsilon$. Otherwise we say $S$ is unstable with respect to (1.1).

We shall use $\langle\cdot, \cdot\rangle$ to denote the pairing of $H^{-2}(\mathbb{R})$ with $H^{2}(\mathbb{R})$ and we remark that when both arguments are in $L^{2}(\mathbb{R})$ then this is equivalent to the inner product on $L^{2}(\mathbb{R})$.

\section{Existence of Traveling Waves}

In this section we obtain solutions to the traveling wave equation (1.5) by solving a constrained minimization problem involving the functionals $I_{c}, K \in C^{2}\left(H^{2}(\mathbb{R}), \mathbb{R}\right)$ defined by

$$
\begin{aligned}
I_{c}(u) & =\frac{1}{2} \int_{-\infty}^{\infty}\left|u_{x x}\right|^{2}-b\left|u_{x}\right|^{2}+c|u|^{2} d x \\
K(u) & =\int_{-\infty}^{\infty} F\left(u, u_{x}\right) d x
\end{aligned}
$$


In addition to (1.3) we assume further that there is some $u \in H^{2}(\mathbb{R})$ such that

$$
\int_{-\infty}^{\infty} F\left(u, u_{x}\right) d x>0
$$

and therefore $K(u)$ is positive for some $u \in H^{2}(\mathbb{R})$. To ensure coercivity of $I_{c}$ we restrict ourselves to $c>b_{+}^{2} / 4$ where $b_{+}=\max (b, 0)$.

Lemma 2.1 The functional $I_{c}$ defined in (2.1) is coercive if $c>b_{+}^{2} / 4$.

Proof. For $u \in H^{2}(\mathbb{R})$ we may integrate by parts to find

$$
\begin{aligned}
I_{c}(u) & =\frac{1}{2} \int_{-\infty}^{\infty}\left|u_{x x}\right|^{2}+b u_{x x} u+c|u|^{2} d x \\
& \geq \frac{1}{2}\left(1-\frac{b_{+}}{2 \sqrt{c}}\right) \int_{-\infty}^{\infty}\left|u_{x x}\right|^{2}+c|u|^{2} d x
\end{aligned}
$$

where we have made use of the inequality $u_{x x} u \leq \frac{\varepsilon u_{x x}^{2}}{2}+\frac{u^{2}}{2 \varepsilon}$ with $\varepsilon=c^{-1 / 2}$. It is clear that the latter integral is equivalent to the $H^{2}(\mathbb{R})$ norm if $c>0$.

Using the homogeneity of $F(q, r)$ we obtain the following estimates on $K$.

Lemma 2.2 Let $F(q, r)$ satisfy (1.3) and let $K(u)$ be defined as in (2.1). Then $K$ is locally Lipschitz on $W^{1, p+1}(\mathbb{R})$.

Proof. Since $F$ is homogeneous of degree $p+1$ it follows that

$$
|F(q, r)| \leq C\left(|q|^{p+1}+|r|^{p+1}\right) .
$$

Indeed, first suppose $0<q<r$. Then by $(1.3)$

$$
|F(q, r)|=r^{p+1}|F(q / r, 1)| \leq C r^{p+1} .
$$

Similarly $|F(q, r)| \leq C q^{p+1}$ for $0<r<q$ and (2.3) holds for positive $q$ and $r$. The result for arbitrary $q$ and $r$ follows similarly. Hence for $u \in W^{1, p+1}(\mathbb{R})$ we have

$$
|K(u)| \leq C\left(\|u\|_{L^{p+1}}^{p+1}+\left\|u_{x}\right\|_{L^{p+1}}^{p+1}\right)=C\|u\|_{W^{1, p+1}}^{p+1}
$$

Next, since both $F_{q}$ and $F_{r}$ are homogeneous of degree $p$ we have

$$
|D F(q, r)| \leq C\left(|q|^{p}+|r|^{p}\right) .
$$

Hence, for $u, v \in W^{1, p+1}(\mathbb{R})$

$$
|K(u)-K(v)| \leq \int_{-\infty}^{\infty}|D F(w, \tilde{w})|\left(|u-v|+\left|u_{x}-v_{x}\right|\right) d x
$$


where $w=\lambda u+(1-\lambda) v, \tilde{w}=\lambda u_{x}+(1-\lambda) v_{x}$ for some function $0 \leq \lambda(x) \leq 1$. By Hölder's inequality and (2.4) we have

$$
\begin{aligned}
|K(u)-K(v)| & \leq\|D F(w, \tilde{w})\|_{L^{\frac{p+1}{p}}}\left(\|u-v\|_{L^{p+1}}+\left\|u_{x}-v_{x}\right\|_{L^{p+1}}\right) \\
& \leq C\left(\|u\|_{W^{1, p+1}}^{p}+\|v\|_{W^{1, p+1}}^{p}\right)\|u-v\|_{W^{1, p+1}}
\end{aligned}
$$

and the lemma is proved.

For $c>b_{+}^{2} / 4$ we now define

$$
m_{\lambda}(c)=\inf \left\{I_{c}(\psi): \psi \in H^{2}(\mathbb{R}), K(\psi)=\lambda\right\}
$$

for $\lambda \geq 0$. By the assumptions (1.3), (2.2) on $F$, there exist $\psi \in H^{2}(\mathbb{R})$ such that $K(\psi)=\lambda$ for $\lambda \geq 0$ and by the coercivity of $I_{c}$ it follows that $m_{\lambda}(c)$ is non-negative and satisfies

$$
m_{\lambda}(c)=\lambda^{\frac{2}{p+1}} m_{1}(c)
$$

for all $\lambda \geq 0$. Hence

$$
m_{\lambda}(c)+m_{1-\lambda}(c)>m_{1}(c)
$$

for $\lambda \in(0,1)$. We call $\psi_{k}$ a minimizing sequence if $I_{c}\left(\psi_{k}\right) \rightarrow m_{1}(c)$ and $K\left(\psi_{k}\right) \rightarrow 1$. The following is an application of the Concentration Compactness Lemma [20].

Theorem 2.3 Let $\psi_{k}$ be a minimizing sequence for the pair of functionals $I_{c}, K$ in (2.1) and suppose $F(q, r)$ satisfies (1.3) and (2.2). Then there exists a subsequence $\left\{\psi_{k_{j}}\right\}, y_{j} \in \mathbb{R}$ and $\psi \in H^{2}(\mathbb{R})$ such that $\psi_{k_{j}} \rightarrow \psi$ in $H^{2}(\mathbb{R})$.

Proof. Let $\psi_{k}$ be a minimizing sequence. Then the condition $c>b_{+}^{2} / 4$ implies that $I_{c}$ is coercive and $\psi_{k}$ is bounded in $H^{2}(\mathbb{R})$, so that the sequence of $L^{1}(\mathbb{R})$ functions

$$
\rho_{k}=\left|\partial_{x}^{2} \psi_{k}\right|^{2}+\left|\psi_{k}\right|^{2}
$$

is bounded. After extracting a subsequence we may assume that $L=\lim _{k \rightarrow \infty} \int_{-\infty}^{\infty} \rho_{k} d x$ exists. By normalizing we may assume further that $\int_{-\infty}^{\infty} \rho_{k} d x=L$ for all $k$. By the Concentration Compactness Lemma [20] there are three possibilities:

(i) Compactness: There exist $y_{k} \in \mathbb{R}$ such that for any $\varepsilon>0$ there exists $R(\varepsilon)>0$ such that for all $k$

$$
\int_{\left|x-y_{k}\right| \leq R(\varepsilon)} \rho_{k} d x \geq \int_{-\infty}^{\infty} \rho_{k} d x-\varepsilon
$$

(ii) Vanishing: For every $R>0$

$$
\lim _{k \rightarrow \infty} \sup _{y \in \mathbb{R}} \int_{|x-y| \leq R} \rho_{k} d x=0
$$

(iii) Dichotomy: There exists $l \in(0, L)$ such that for all $\varepsilon>0$ there exist $R, R_{k} \rightarrow \infty$, $y_{k} \in \mathbb{R}$ and $k_{0}$ such that

$$
\left|\int_{\left|x-y_{k}\right| \leq R} \rho_{k} d x-l\right|<\varepsilon \quad \text { and } \quad\left|\int_{R<\left|x-y_{k}\right|<R_{k}} \rho_{k} d x\right|<\varepsilon
$$


for $k>k_{0}$.

We first show that compactness of $\rho_{k}$ implies the existence of a minimizer. Since $\psi_{k}$ is bounded in $H^{2}(\mathbb{R})$ there is some subsequence $\psi_{j}$ and some $\psi \in H^{2}(\mathbb{R})$ such that

$$
\begin{array}{lll}
\psi_{j}\left(\cdot+y_{j}\right) \rightarrow \psi & \text { in } & H^{2}(\mathbb{R}) \\
\psi_{j}\left(\cdot+y_{j}\right) \rightarrow \psi & \text { in } & H_{l o c}^{1}(\mathbb{R})
\end{array}
$$

Also since $\psi_{j}$ is bounded in $W^{1, q}(\mathbb{R})$ for all $q$, the convergence also takes place strongly in $W_{l o c}^{1, p+1}(\mathbb{R})$. By compactness of $\rho_{k}$ and the Sobolev inequality it follows that the sequence

$$
\sigma_{j}=\left|\partial_{x} \psi_{j}\left(\cdot+y_{j}\right)\right|^{p+1}+\left|\psi_{j}\left(\cdot+y_{j}\right)\right|^{p+1}
$$

is also compact. We claim this implies strong convergence in $W^{1, p+1}(\mathbb{R})$. Given $\varepsilon>0$ choose $R_{0}$ so large that

$$
\int_{|x| \geq R_{0}}\left|\partial_{x} \psi\right|^{p+1}+|\psi|^{p+1} d x<\varepsilon
$$

By compactness of $\sigma_{j}$ there exist $j_{1}(\varepsilon)$ and $R(\varepsilon)>R_{0}$ such that

$$
\int_{|x| \geq R(\varepsilon)} \sigma_{j} d x<\varepsilon
$$

for $j \geq j_{1}(\varepsilon)$. By the convergence in $W_{l o c}^{1, p+1}(\mathbb{R})$ there exists $j_{2}(\varepsilon) \geq j_{1}(\varepsilon)$ such that

$$
\left\|\psi_{j}\left(\cdot+y_{j}\right)-\psi\right\|_{W^{1, p+1}(B(0, R(\varepsilon)))}^{p+1}<\varepsilon
$$

for $j \geq j_{2}(\varepsilon)$. Thus

$$
\left\|\psi_{j}\left(\cdot+y_{j}\right)-\psi\right\|_{W^{1, p+1}(\mathbb{R})}<\left(2^{p+1}+1\right) \varepsilon
$$

for $j \geq j_{2}(\varepsilon)$, which proves the claim. Thus by Lemma 2.2 it follows that $K(\psi)=1$. By the weak convergence in $H^{2}(\mathbb{R})$ and the weak lower semicontinuity of $I_{c}$ we have $I_{c}(\psi) \leq m_{1}(c)$, and therefore $\psi$ must be a minimizer.

We next prove that $\rho_{k}$ is compact by ruling out possibilities (ii) and (iii). First suppose that (ii) holds. It follows from (2.3) and the Sobolev inequality that

$$
\int_{|x-y| \leq 1} F\left(\psi_{k}, \partial_{x} \psi_{k}\right) d x \leq C\left(\int_{|x-y| \leq 1} \rho_{k} d x\right)^{\frac{p+1}{2}}
$$

for all $y \in \mathbb{R}$. By (ii) we can choose $k(\varepsilon)$ so large that

$$
\int_{|x-y| \leq 1} F\left(\psi_{k}, \partial_{x} \psi_{k}\right) d x \leq C \varepsilon^{\frac{p-1}{2}} \int_{|x-y| \leq 1} \rho_{k} d x
$$

for $k \geq k(\varepsilon)$. Summing over intervals centered at even integers gives

$$
K\left(\psi_{k}\right) \leq C \varepsilon^{\frac{p-1}{2}}
$$

and we arrive at the contradiction that $K\left(\psi_{k}\right) \rightarrow 0$ as $k \rightarrow \infty$. Next suppose (iii) holds. Then we may define cutoff functions $\xi_{1}$ and $\xi_{2}$ with support on $|x| \leq 2$ and $|x| \geq 1 / 2$ 
respectively and with $\xi_{1}(x)=1$ for $|x| \leq 1$ and $\xi_{2}(x)=1$ for $|x| \geq 1$, in such a way that the functions $\psi_{k, 1}(x)=\xi_{1}\left(\left|x-y_{k}\right| / R\right) \psi_{k}(x)$ and $\psi_{k, 2}(x)=\xi_{2}\left(\left|x-y_{k}\right| / R_{k}\right) \psi_{k}(x)$ satisfy

$$
\begin{aligned}
& I_{c}\left(\psi_{k}\right)=I_{c}\left(\psi_{k, 1}\right)+I_{c}\left(\psi_{k, 2}\right)+O(\varepsilon) \\
& K\left(\psi_{k}\right)=K\left(\psi_{k, 1}\right)+K\left(\psi_{k, 2}\right)+O(\varepsilon)
\end{aligned}
$$

for $k \geq k_{0}$. Since $\left\{\psi_{k}\right\}_{k=1}^{\infty}$ is bounded in $H^{2}(\mathbb{R})$ it follows that $\psi_{k, 1}$ and $\psi_{k, 2}$ are also bounded in $H^{2}(\mathbb{R})$ independently of $\varepsilon$. Hence $K\left(\psi_{k, 1}\right)$ and $K\left(\psi_{k, 2}\right)$ are bounded and we may pass to a subsequence to define $\lambda_{i}(\varepsilon)=\lim _{k \rightarrow \infty} K\left(\psi_{k, i}\right)$ for $i=1$, 2. Since $\lambda_{1}(\varepsilon)$ and $\lambda_{2}(\varepsilon)$ are bounded independently of $\varepsilon$ we can choose a sequence $\varepsilon_{j} \rightarrow 0$ such that the limits $\lambda_{i}=\lim _{j \rightarrow \infty} \lambda_{i}\left(\varepsilon_{j}\right)$ exist. We clearly have $\lambda_{1}+\lambda_{2}=1$ and there are three cases to consider.

If $\lambda_{1} \in(0,1)$ then by $(2.6)$

$$
\begin{aligned}
I_{c}\left(\psi_{k}\right) & =I_{c}\left(\psi_{k, 1}\right)+I_{c}\left(\psi_{k, 2}\right)+O\left(\varepsilon_{j}\right) \\
& \geq m_{K\left(\psi_{k, 1}\right)}(c)+m_{K\left(\psi_{k, 2}\right)}(c)+O\left(\varepsilon_{j}\right) \\
& =\left(K\left(\psi_{k, 1}\right)^{\frac{2}{p+1}}+K\left(\psi_{k, 2}\right)^{\frac{2}{p+1}}\right) m_{1}(c)+O\left(\varepsilon_{j}\right)
\end{aligned}
$$

Letting $k \rightarrow \infty$ and using the fact that $\psi_{k}$ is a minimizing sequence yields

$$
m_{1}(c) \geq\left(\lambda_{1}\left(\varepsilon_{j}\right)^{\frac{2}{p+1}}+\lambda_{2}\left(\varepsilon_{j}\right)^{\frac{2}{p+1}}\right) m_{1}(c)+O\left(\varepsilon_{j}\right)
$$

and letting $j \rightarrow \infty$ we arrive at the contradiction

$$
m_{1}(c) \geq\left(\lambda_{1}^{\frac{2}{p+1}}+\lambda_{2}^{\frac{2}{p+1}}\right) m_{1}(c)>m_{1}(c) .
$$

If $\lambda_{1}=0$ (or equivalently $\lambda_{1}=1$ ) we use the coercivity of $I_{c}$ and the assumption of dichotomy to estimate

$$
\begin{aligned}
I_{c}\left(\psi_{k, 1}\right) & \geq \frac{1}{2}\left(1-\sqrt{b_{+}^{2} / 4 c}\right) \int_{-\infty}^{\infty}\left|\partial_{x}^{2} \psi_{k, 1}\right|^{2}+\left|\psi_{k, 1}\right|^{2} d x \\
& =\frac{1}{2}\left(1-\sqrt{b_{+}^{2} / 4 c}\right) \int_{\left.\left|x-y_{k}\right| \leq 2 R\right)}\left|\partial_{x}^{2} \psi_{k}\right|^{2}+\left|\psi_{k}\right|^{2} d x+O\left(\varepsilon_{j}\right) \\
& =\frac{1}{2}\left(1-\sqrt{b_{+}^{2} / 4 c}\right)\left(l+O\left(\varepsilon_{j}\right)\right) .
\end{aligned}
$$

Thus

$$
\begin{aligned}
I_{c}\left(\psi_{k}\right) & =I_{c}\left(\psi_{k, 1}\right)+I_{c}\left(\psi_{k, 2}\right)+O\left(\varepsilon_{j}\right) \\
& \geq \frac{1}{2}\left(1-\sqrt{b_{+}^{2} / 4 c}\right)\left(l+O\left(\varepsilon_{j}\right)\right)+K\left(\psi_{k, 2}\right)^{\frac{2}{p+1}} m_{1}(c)+O\left(\varepsilon_{j}\right) .
\end{aligned}
$$

As above we let $k \rightarrow \infty$ and then $j \rightarrow \infty$ to find

$$
m_{1}(c) \geq \frac{1}{2}\left(1-\sqrt{b_{+}^{2} / 4 c}\right) l+m_{1}(c)>m_{1}(c)
$$


a contradiction.

Finally, if $\lambda_{1}>1$ (or equivalently $\lambda_{1}<0$ ) we use the positivity of $I_{c}$ to estimate

$$
I_{c}\left(\psi_{k}\right) \geq I_{c}\left(\psi_{k, 1}\right)+O\left(\varepsilon_{j}\right) \geq K\left(\psi_{k, 1}\right)^{\frac{2}{p+1}} m_{1}(c)+O\left(\varepsilon_{j}\right)
$$

Once again sending $k, j \rightarrow \infty$ yields the contradiction

$$
m_{1}(c) \geq \lambda_{1}^{\frac{2}{p+1}} m_{1}(c)>m_{1}(c) .
$$

Hence the sequence $\rho_{k}$ is compact.

The function $\psi$ found in Theorem 2.3 is a minimizer of $I_{c}$ subject to the constraint $K=1$ and is therefore a weak solution of the Euler-Lagrange equation

$$
\psi_{x x x x}+b \psi_{x x}+c \psi=\mu f\left(\psi, \psi_{x}, \psi_{x x}\right)
$$

for some multiplier $\mu$. By the homogeneity of $f$ we can rescale to obtain a solution $\varphi=\mu^{\frac{1}{p-1}} \psi$ of (1.5). Such a solution will be called a ground state. We now show that this weak solution is in fact a classical solution of (1.5).

Lemma 2.4 Suppose $\varphi \in H^{2}(\mathbb{R})$ is a weak solution of $(1.5)$. Then $\varphi$ is a classical solution and $\varphi \in C^{5}(\mathbb{R})$. Furthermore, $|\varphi| \rightarrow 0$ exponentially as $x \rightarrow \pm \infty$.

Proof. By (1.2) and (1.3) we have

$$
|f(q, r, s)| \leq C\left(|q|^{p}+|r|^{p}+|r||q|^{p-1}+|s|\left(|q|^{p-1}+|r|^{p-1}\right)\right)
$$

Since $\varphi \in H^{2}(\mathbb{R})$, both $\varphi$ and $\varphi_{x}$ are in $L^{\infty}(\mathbb{R}) \cap L^{2}(\mathbb{R})$ and thus $f\left(\varphi, \varphi_{x}, \varphi_{x x}\right) \in L^{2}(\mathbb{R})$. Since $\varphi$ is a weak solution of (1.5) this imples $\varphi \in H^{4}(\mathbb{R})$ and therefore $f\left(\varphi, \varphi_{x}, \varphi_{x x}\right) \in$ $C^{1}(\mathbb{R})$. Thus $\varphi \in C^{5}(\mathbb{R})$ is a classical solution of (1.5). Next we write (1.5) as a system $\phi^{\prime}=A \phi+P(\phi)$ where

$$
A=\left[\begin{array}{cccc}
0 & 1 & 0 & 0 \\
0 & 0 & 1 & 0 \\
0 & 0 & 0 & 1 \\
-c & 0 & -b & 0
\end{array}\right]
$$

and $P\left(\phi_{1}, \phi_{2}, \phi_{3}, \phi_{4}\right)=\left(0,0,0, f\left(\phi_{1}, \phi_{2}, \phi_{3}\right)\right)$. It follows from the condition $c>b_{+}^{2}$ that $A$ has two eigenvalues with positive real part and two with negative real part. Thus the exponential decay follows from the stable-manifold theorem if we can show that $\varphi(x)$ and its first three derivatives approach zero as $x \rightarrow \infty$. But this follows from

$$
\|\varphi\|_{C^{3}([n, n+1])} \leq C\|\varphi\|_{H^{4}([n, n+1])} \rightarrow 0 \quad \text { as } \quad n \rightarrow \infty
$$

since $\varphi \in H^{4}(\mathbb{R})$.

Remark 2.5 At present it is not known whether the ground state solutions of (1.5) are unique, up to translations and symmetries of the nonlinear term. Thus we consider the stability of the set of ground states $\mathcal{G}_{c}$. See (3.6) below. 


\section{The function $d(c)$}

The functionals $E$ and $Q$ are related to the functionals $I_{c}$ and $K$ used to obtain the traveling waves by the simple formula

$$
E(u)+c Q(u)=I_{c}(u)-K(u) .
$$

It is precisely this relationship that makes it possible to utilize the variational properties of the traveling waves in the stability analysis. In light of (1.8) and (3.1) we define $d(c)$ by

$$
d(c)=E(\varphi)+c Q(\varphi),
$$

for $c>b_{+}^{2} / 4$, where $\varphi$ is any ground state solution of (1.5).

Lemma 3.1 The function $d(c)$ is well defined on $\left(b_{+}^{2} / 4, \infty\right)$, is strictly increasing, and is differentiable at all but countably many points.

Proof. First we notice that by the homogeneity of $F$, any ground state is a minimizer of the quotient

$$
\frac{I_{c}(u)}{K(u)^{2 / p+1}}
$$

over non-zero $u \in H^{2}(\mathbb{R})$. Thus, multiplying (1.5) by $\varphi$ and using (1.3) we find that

$$
2 I_{c}(\varphi)=(p+1) K(\varphi)=(p+1)\left(\frac{2 m_{1}(c)}{p+1}\right)^{\frac{p+1}{p-1}} .
$$

This shows that $d(c)$ is well defined and

$$
d(c)=\frac{p-1}{p+1} I_{c}(\varphi)=\frac{p-1}{2} K(\varphi)=\frac{p-1}{2}\left(\frac{2 m_{1}(c)}{p+1}\right)^{\frac{p+1}{p-1}} .
$$

Next, for $0 \neq u \in H^{2}(\mathbb{R})$ fixed, $L_{u}(c)=I_{c}(u) / K(u)^{2 / p+1}$ is a line. Since $m_{1}(c)$ is the infimum of this family of lines, it follows that $m_{1}(c)$ is a concave function on $\left(b_{+}^{2} / 4, \infty\right)$, and thus is continuous and differentiable at all but countably many points. By (3.4) the same regularity properties hold for $d(c)$. Next let $\varphi_{1}$ and $\varphi_{2}$ be ground states with speeds $c_{1}$ and $c_{2}$ respectively, with $c_{1}>c_{2}$. Then

$$
m_{1}\left(c_{1}\right)=\frac{I_{c_{1}}\left(\varphi_{1}\right)}{K\left(\varphi_{1}\right)^{2 / p+1}}=\frac{I_{c_{2}}\left(\varphi_{1}\right)+\frac{1}{2}\left(c_{1}-c_{2}\right) \int_{\mathbb{R}} \varphi_{1}^{2} d x}{K\left(\varphi_{1}\right)^{2 / p+1}} \geq m_{1}\left(c_{2}\right)+\frac{\left(c_{1}-c_{2}\right) \int_{\mathbb{R}} \varphi_{1}^{2} d x}{2 K\left(\varphi_{1}\right)^{2 / p+1}}
$$

This shows that $m_{1}$ is strictly increasing, so that by (3.4) $d$ must be strictly increasing as well.

In view of (3.4) we define the set of ground states with speed $c$ by

$$
\mathcal{G}_{c}=\left\{\varphi \in H^{2}(\mathbb{R}): 2(p-1) I_{c}(\varphi)=\left(p^{2}-1\right) K(\varphi)=2(p+1) d(c)\right\}
$$

for $c>b_{+}^{2} / 4$. 
Lemma 3.2 At points of differentiability of $d(c)$ we have

$$
d^{\prime}(c)=Q(\varphi)
$$

for any $\varphi \in \mathcal{G}_{c}$.

Proof. Again let $c_{1}>c_{2}$. By reversing the roles of $c_{1}$ and $c_{2}$ in (3.5) we find that

$$
\frac{\int_{\mathbb{R}} \varphi_{1}^{2} d x}{2 K\left(\varphi_{1}\right)^{2 / p+1}} \leq \frac{m\left(c_{1}\right)-m\left(c_{2}\right)}{c_{1}-c_{2}} \leq \frac{\int_{\mathbb{R}} \varphi_{2}^{2} d x}{2 K\left(\varphi_{2}\right)^{2 / p+1}}
$$

Because this holds for all such ground states, we have also that

$$
\frac{q_{s}\left(c_{1}\right)}{\left(\frac{2 m_{1}\left(c_{1}\right)}{p+1}\right)^{2 / p-1}} \leq \frac{m\left(c_{1}\right)-m\left(c_{2}\right)}{c_{1}-c_{2}} \leq \frac{q_{i}\left(c_{2}\right)}{\left(\frac{2 m_{1}\left(c_{2}\right)}{p+1}\right)^{2 / p-1}}
$$

where $q_{i}(c)$ and $q_{s}(c)$ are the infimum and supremum, respectively, of $\left\{Q(\varphi): \varphi \in \mathcal{G}_{c}\right\}$. We claim that $\lim \sup _{c \rightarrow c_{0}} q_{i}(c) \leq q_{s}\left(c_{0}\right)$ and $\liminf _{c \rightarrow c_{0}} q_{s}(c) \geq q_{i}\left(c_{0}\right)$. It follows from the claim and (3.4) that the right and left derivatives of $d(c)$ are $d^{\prime}\left(c^{+}\right)=q_{i}(c)$ and $d^{\prime}\left(c^{-}\right)=q_{s}(c)$, respectively. At points of differentiability we have $q_{i}(c)=q_{s}(c)$ and the lemma follows. To prove the claim let $c_{k} \rightarrow c_{0}$ and choose $\varphi_{k} \in \mathcal{G}_{c_{k}}$. By (3.3) and the continuity of $m_{1}$ it follows that $\varphi_{k}$ is a minimizing sequence for the pair $I_{c_{0}}, K$. Thus by Theorem 2.3 some subsequence $\varphi_{k_{j}}$ when translated appropriately, converges to some $\varphi_{0} \in \mathcal{G}_{c_{0}}$. Thus $\limsup _{j \rightarrow \infty} q_{i}\left(c_{k_{j}}\right) \leq \lim _{j \rightarrow \infty} Q\left(\varphi_{k_{j}}\right)=Q\left(\varphi_{0}\right) \leq q_{s}\left(c_{0}\right)$. Similarly liminf $\operatorname{in}_{j \rightarrow \infty} q_{s}\left(c_{k_{j}}\right) \leq q_{i}\left(c_{0}\right)$. Since this holds for all sequences $c_{k} \rightarrow c_{0}$, this proves the claim.

Lemma 3.3 Suppose $b=0$ and $F(q, r)$ is homogeneous in $r$ of degree $\beta$. Then for all $c>0$ we have

$$
d(c)=d(1) c^{\gamma} \quad \text { where } \quad \gamma=\frac{3 p-2 \beta+5}{4(p-1)}
$$

Proof. It follows from the homogeneity of $F$ in $r$ that if we set $v(x)=\lambda u(\mu c)$ for $\lambda, \mu>0$, then $f\left(v, v_{v}, v_{x x}\right)=\lambda^{p} \mu^{\beta} f\left(u, u_{x}, u_{x x}\right)$. Thus if $\varphi_{1}$ is a solution of (1.5) with $c=1$ it follows that

$$
\varphi_{c}(x)=c^{(4-\beta) / 4(p-1)} \varphi_{1}\left(c^{1 / 4} x\right)
$$

is a solution of (1.5) with speed $c$. The lemma then follows from (3.4) by computing $K\left(\varphi_{c}\right)$.

\section{Stability}

Our main result in this section is the following.

Theorem 4.1 Let $c>b_{+}^{2} / 4$ and suppose Assumption 1.1 holds. If $d^{\prime \prime}(c)>0$ then the set of ground states $\mathcal{G}_{c}$ is stable. 
Remark 4.2 For speeds $c$ at which $d$ is not twice differentiable, we may replace the condition $d^{\prime \prime}(c)>0$ by strict convexity in an interval containing c. See [26], [19].

Let

$$
U_{c, \varepsilon}=\left\{v \in H^{2}(\mathbb{R}): \inf \left\{\|v-\varphi\|_{H^{2}(\mathbb{R})}: \varphi \in \mathcal{G}_{c}\right\}<\varepsilon\right\}
$$

denote the $\varepsilon$-neighborhood around $\mathcal{G}_{c}$. Since $d(c)$ is strictly increasing, we may associate to any $u \in U_{c, \varepsilon}$ the speed

$$
c(u)=d^{-1}\left(\frac{p-1}{2} K(u)\right) .
$$

The following lemma provides the crucial bound involving these speeds.

Lemma 4.3 If $d^{\prime \prime}(c)>0$ then there exists $\varepsilon>0$ such that for any $u \in U_{c, \varepsilon}$ and $\varphi \in \mathcal{G}_{c}$ we have

$$
E(u)-E(\varphi)+c(u)(Q(u)-Q(\varphi)) \geq \frac{1}{4} d^{\prime \prime}(c)|c(u)-c|^{2}
$$

Proof. Since $d^{\prime}(c)=Q(\varphi)$ we have the expansion

$$
d(\tilde{c})=d(c)+Q(\varphi)(\tilde{c}-c)+\frac{1}{2} d^{\prime \prime}(c)(\tilde{c}-c)^{2}+o\left(|\tilde{c}-c|^{2}\right)
$$

for $\tilde{c}$ near $c$. By choosing $\varepsilon$ sufficiently small the continuity of $c(u)$ implies that

$$
\begin{aligned}
d(c(u)) & \geq d(c)+Q(\varphi)(c(u)-c)+\frac{1}{4} d^{\prime \prime}(c)(c(u)-c)^{2} \\
& =E(\varphi)+c(u) Q(\varphi)+\frac{1}{4} d^{\prime \prime}(c)(c(u)-c)^{2}
\end{aligned}
$$

Next, if $\varphi_{c(u)} \in S_{c(u)}$ then, by (3.4) and (4.1), $K\left(\varphi_{c(u)}\right)=\frac{2}{p-1} d(c(u))=K(u)$. Since $\varphi_{c(u)}$ minimizes $I_{c}$ over all $u$ with $K(u)=\frac{2}{p-1} d(c(u))$ we then have

$$
\begin{aligned}
E(u)+c(u) Q(u) & =I_{c(u)}(u)-K(u) \\
& \geq I_{c(u)}\left(\varphi_{c(u)}\right)-K\left(\varphi_{c(u)}\right) \\
& =d(c(u))
\end{aligned}
$$

which proves the lemma.

Proof of Theorem 4.1 Suppose $\mathcal{G}_{c}$ is unstable and choose initial data $g_{k}$ so that

$$
\inf _{\varphi \in \mathcal{G}_{c}}\left\|g_{k}-\varphi\right\|_{H^{2}(\mathbb{R})}<\frac{1}{k}
$$

and let $u_{k}(t)$ be the solution of (1.1) with $u_{k}(0)=g_{k}$. Then, by Assumption $1.1 u_{k}$ is continuous in $t$, and there exist $t_{k}$ so that

$$
\inf _{\varphi \in \mathcal{G}_{c}}\left\|u_{k}\left(t_{k}\right)-\varphi\right\|_{H^{2}(\mathbb{R})}=\delta .
$$


Since $E$ and $Q$ are invariants of (1.1) and since $\mathcal{G}_{c}$ is bounded, we can find $\varphi_{k} \in \mathcal{G}_{c}$ so that

$$
\begin{aligned}
& E\left(u_{k}\left(t_{k}\right)\right)-E\left(\varphi_{k}\right) \rightarrow 0 \\
& Q\left(u_{k}\left(t_{k}\right)\right)-Q\left(\varphi_{k}\right) \rightarrow 0 .
\end{aligned}
$$

as $k \rightarrow \infty$. If $\delta$ is chosen so small that Lemma 4.3 applies, then

$$
E\left(u_{k}\left(t_{k}\right)\right)-E\left(\varphi_{k}\right)+c\left(u_{k}\left(t_{k}\right)\right)\left[Q\left(u_{k}\left(t_{k}\right)\right)-Q\left(\varphi_{k}\right)\right] \geq \frac{1}{4} d^{\prime \prime}(c)\left|c\left(u_{k}\left(t_{k}\right)\right)-c\right|^{2}
$$

and therefore, by (4.3), $c\left(u_{k}\left(t_{k}\right)\right) \rightarrow c$ as $k \rightarrow \infty$. The continuity of $d$ then implies that

$$
K\left(u_{k}\left(t_{k}\right)\right) \rightarrow \frac{2}{p-1} d(c)
$$

which in turn, using (3.1), (4.3) and (3.2), implies that

$$
I_{c}\left(u_{k}\left(t_{k}\right)\right) \rightarrow \frac{2(p+1)}{p-1} d(c) .
$$

Hence $u_{k}\left(t_{k}\right)$ is a minimizing sequence for the pair $I_{c}, K$ and thus by Theorem 2.3 has a subsequence $u_{k_{j}}\left(t_{k_{j}}\right)$ such that

$$
\lim _{j \rightarrow \infty}\left\|u_{k_{j}}\left(t_{k_{j}}\right)-\varphi_{j}\right\|_{H^{2}(\mathbb{R})}=0
$$

for some sequence $\left\{\varphi_{j}\right\}_{j=1}^{\infty} \subset \mathcal{G}_{c}$. Since this contradicts (4.2) the theorem is proved.

\section{$5 \quad$ Instability}

Our main assumption in this section is the following.

Assumption 5.1 There exists a choice $\varphi(c)$ which is $C^{1}$ as a map from $\left(b_{+}^{2} / 4, \infty\right)$ to $H^{2}(\mathbb{R})$ such that $\varphi(c) \in \mathcal{G}_{c}$.

Remark 5.2 As shown in the proof of Lemma 3.3, Assumption 5.1 holds with the map given by (3.8) whenever $b=0$ and $F(q, r)$ is jointly homogeneous in $q$ and $r$.

We will denote by $\varphi^{\prime}(c)$ the derivative of $\varphi(c)$ with respect to the parameter $c$. We define $T(r), r \in \mathbb{R}$ to be the group of translations acting on $H^{2}(\mathbb{R})$ by

$$
T(r) u(x)=u(x+r) .
$$

For $c>b_{+}^{2} / 4$ the orbit of the traveling wave $\varphi(c)$ is given by

$$
\mathcal{T}_{c}=\{T(r) \varphi(c): r \in \mathbb{R}\}
$$

and we define

$$
V_{c, \varepsilon}=\left\{v \in H^{2}(\mathbb{R}): \inf \left\{\|\psi-v\|_{H^{2}(\mathbb{R})}: \psi \in \mathcal{T}_{c}\right\}<\varepsilon\right\}
$$

to be the $\varepsilon$-neighborhood of $\mathcal{T}_{c}$. 
Theorem 5.3 Suppose Assumptions 5.1 and 1.1 hold. Let $c>b_{+}^{2} / 4$ and $p \geq 2$. If $d^{\prime \prime}(c)<0$ then $\mathcal{T}_{c}$ is unstable.

We first prove a result concerning the regularity of $\varphi^{\prime}(c)$.

Lemma 5.4 Suppose $c>b_{+}^{2} / 4$ and Assumption 5.1 holds. Then $\varphi^{\prime}(c) \in C^{4}(\mathbb{R})$ and $\left|\varphi^{\prime}(c)\right| \rightarrow 0$ exponentially as $x \rightarrow \pm \infty$.

Proof. Since $\varphi(c)$ is a weak solution of (1.5) it follows by differentiating with respect to $c$ that $\varphi^{\prime}(c)$ is a weak solution of

$$
u_{x x x x}+b u_{x x}+c u=-\varphi+f_{q} u+f_{r} u_{x}+f_{s} u_{x x}
$$

where $f_{q}=F_{q q}\left(\varphi, \varphi_{x}\right)-\varphi_{x} F_{q q r}\left(\varphi, \varphi_{x}\right)-\varphi_{x x} F_{q r r}\left(\varphi, \varphi_{x}\right), f_{r}=-\varphi_{x} F_{q r r}\left(\varphi, \varphi_{x}\right)-\varphi_{x x} F_{r r r}\left(\varphi, \varphi_{x}\right)$ and $f_{s}=-F_{r r}\left(\varphi, \varphi_{x}\right)$, and we have suppressed the dependence on $c$. Since both $\varphi(c), \varphi^{\prime}(c) \in$ $H^{2}(\mathbb{R})$ and $F \in C^{3}\left(\mathbb{R}^{2}\right)$, it follows that each term on the right hand side is in $L^{2}(\mathbb{R})$. Hence $\varphi^{\prime}(c) \in H^{4}(\mathbb{R})$ and the right hand side is continuous, so that $\varphi^{\prime}(c) \in C^{4}(\mathbb{R})$. The exponential decay follows as in Lemma 2.4.

The following lemmas supply the tools with which to constuct a Lyapunov functional. Notice that, if

$$
H_{c}=E^{\prime \prime}(\varphi(c))+c Q^{\prime \prime}(\varphi(c))
$$

denotes the operator associated with the linearization around $\varphi(c)$, we have

$$
\left\langle H_{c} \varphi(c), \varphi(c)\right\rangle=-\left(p^{2}-1\right) K(\varphi(c))<0
$$

Thus the ground state itself may be used to produce an unstable direction.

Lemma 5.5 If $d^{\prime \prime}(c)<0$ then there exists $y \in H^{2}(\mathbb{R})$ so that

$$
\begin{aligned}
\text { (i) } & \left\langle H_{c} y, y\right\rangle<0 \\
\text { (ii) } & \langle\varphi(c), y\rangle=0 .
\end{aligned}
$$

Proof. Define

$$
q(h, \sigma)=Q(\varphi(h)+\sigma \varphi(c)) .
$$

Then

$$
q(c, 0)=Q(\varphi(c))=d^{\prime}(c)
$$

and

$$
\frac{\partial q}{\partial h}(c, 0)=\left\langle Q^{\prime}(\varphi(c)), \varphi^{\prime}(c)\right\rangle=d^{\prime \prime}(c)<0
$$

The Implicit Function Theorem then implies that there exists $\varepsilon>0$ and a $C^{2}$ map $h$ : $(-\varepsilon, \varepsilon) \rightarrow\left(b_{+}^{2} / 4, \infty\right)$ such that

$$
q(h(\sigma), \sigma)=Q(\varphi(h(\sigma))+\sigma \varphi(c))=Q(\varphi(c)) \quad \text { and } \quad h(0)=c .
$$


If we define

$$
y=h^{\prime}(0) \varphi^{\prime}(c)+\varphi(c)
$$

then $y \in H^{2}(\mathbb{R})$ by Assumption 5.1, and we have

$$
\langle\varphi(c), y\rangle=\left\langle Q^{\prime}(\varphi(c)), y\right\rangle=\frac{d}{d \sigma} q(h(\sigma), \sigma)=0
$$

and thus (ii) is satisfied. To show that (i) holds we define

$$
\psi(\sigma)=\varphi(h(\sigma))+\sigma \varphi(c)
$$

and set

$$
\mathcal{E}(\sigma)=E(\psi(\sigma))
$$

It follows from (5.5) and (1.8) that We next show that

$$
\begin{aligned}
\mathcal{E}(0) & =E(\varphi(c)) \\
\mathcal{E}^{\prime}(0) & =0 \\
\mathcal{E}^{\prime \prime}(0) & =\left\langle H_{c} y, y\right\rangle .
\end{aligned}
$$

The first statement is obvious. Next we compute

$$
\begin{aligned}
\mathcal{E}^{\prime}(\sigma) & =\left\langle E^{\prime}(\psi(\sigma)), \psi(\sigma)\right\rangle \\
0 & =c\left\langle Q^{\prime}(\psi(\sigma)), \psi(\sigma)\right\rangle
\end{aligned}
$$

Adding these equations, evaluating at $\sigma=0$, and using the fact that $\varphi(c)$ satisfies (1.8) yields the second statement. For the third assertion we compute

$$
\begin{aligned}
\mathcal{E}^{\prime \prime}(\sigma) & =\left\langle E^{\prime \prime}(\psi(\sigma)) \psi^{\prime}(\sigma), \psi^{\prime}(\sigma)\right\rangle+\left\langle E^{\prime}(\psi(\sigma)), \psi^{\prime \prime}(\sigma)\right\rangle \\
0 & =c\left\langle Q^{\prime \prime}(\psi(\sigma)) \psi^{\prime}(\sigma), \psi^{\prime}(\sigma)\right\rangle+c\left\langle Q^{\prime}(\psi(\sigma)), \psi^{\prime \prime}(\sigma)\right\rangle
\end{aligned}
$$

If we add these equations and evaluate at $\sigma=0$ then the first order terms again cancel so that we are left with

$$
\mathcal{E}^{\prime \prime}(0)=\left\langle E^{\prime \prime}(\varphi(c))+c Q^{\prime \prime}(\varphi(c)) \psi^{\prime}(0), \psi^{\prime}(0)\right\rangle=\left\langle H_{c} y, y\right\rangle .
$$

With (5.8) established we now need to consider the second order Taylor expansions of $E$ and $Q$ at $\varphi(h(\sigma))$. We first write

$$
\begin{aligned}
& E(u+v)=E(u)+\left\langle E^{\prime}(u), v\right\rangle+\frac{1}{2}\left\langle E^{\prime \prime}(u) v, v\right\rangle+o\left(\|v\|^{2}\right) \\
& Q(u+v)=Q(u)+\left\langle Q^{\prime}(u), v\right\rangle+\frac{1}{2}\left\langle Q^{\prime \prime}(u) v, v\right\rangle+o\left(\|v\|^{2}\right)
\end{aligned}
$$

With $u=\varphi(h(\sigma))$ and $v=\sigma \varphi(c)$ we have $\psi(\sigma)=u+v$ and

$$
E(\psi(\sigma))+h(\sigma) Q(\psi(\sigma))=d(h(\sigma))+\frac{1}{2} \sigma^{2}\left\langle H_{h(\sigma)} \varphi(c), \varphi(c)\right\rangle+o\left(\sigma^{2}\right)
$$


where we have made use of (3.2), (1.8) and (5.1) with $c=h(\sigma)$. Hence, by (5.3) and (5.7) we have

$$
\mathcal{E}(\sigma)=\frac{1}{2} \sigma^{2}\left\langle H_{h(\sigma)} \varphi(c), \varphi(c)\right\rangle-h(\sigma) Q(\varphi(c))+d(h(\sigma))+o\left(\sigma^{2}\right)
$$

By the concavity of $d$ and (3.7) we have

$$
d(h(\sigma)) \leq d(c)+(h(\sigma)-c) d^{\prime}(c)=E(\varphi(c))+h(\sigma) Q(\varphi(c))
$$

so that

$$
\begin{aligned}
\mathcal{E}(\sigma) & \leq \frac{1}{2} \sigma^{2}\left\langle H_{h(\sigma)} \varphi(c), \varphi(c)\right\rangle+E(\varphi(c))+o\left(\sigma^{2}\right) \\
& <\frac{1}{4} \sigma^{2}\left\langle H_{c} \varphi(c), \varphi(c)\right\rangle+E(\varphi(c))+o\left(\sigma^{2}\right)
\end{aligned}
$$

for $\sigma$ small enough. Together with (5.8) this implies that $\mathcal{E}^{\prime \prime}(0)<0$ and therefore (i) holds.

We omit the proof of the following lemma, which is the analogue of ([7] Lemma 4.1).

Lemma 5.6 There exist $\varepsilon>0$ and $a C^{1}$ map $s: V_{c, \varepsilon} \rightarrow \mathbb{R}$ so that for $v \in V_{c, \varepsilon}$ and $r \in \mathbb{R}$ we have

$$
\begin{aligned}
(i) & s(\varphi(c))=0 \\
(\text { ii }) & \left\langle T(s(v)) v, \partial_{x} \varphi(c)\right\rangle=0 \\
(\text { iii }) & s(T(r) v)=s(v)-r \\
(\text { iv }) & s^{\prime}(v)=\frac{T(-s(v)) \partial_{x} \varphi(c)}{\int_{-\infty}^{\infty} v(x) T(-s(v)) \partial_{x}^{2} \varphi(c) d x}
\end{aligned}
$$

We now define

$$
\begin{aligned}
B(v) & =T(-s(v)) y-\langle v, T(-s(v)) y\rangle \partial_{x} s^{\prime}(v) \\
& =T(-s(v)) y-\frac{\langle v, T(-s(v)) y\rangle}{\left\langle v, T(-s(v)) \partial_{x}^{2} \varphi(c)\right\rangle} T(-s(v)) \partial_{x}^{2} \varphi(c)
\end{aligned}
$$

Lemma $5.7 B$ is a $C^{1}$ function from $V_{c, \varepsilon}$ to $H^{2}(\mathbb{R})$ which commutes with $T$ and satisfies $B(\varphi(c))=y$ and $\langle B(v), v\rangle=0$ for all $v \in V_{c, \varepsilon}$.

Proof. That $B$ is $C^{1}$ follows from the fact that $y$ is a linear combination of $\varphi(c)$ and $\varphi^{\prime}(c)$, and both of these are in $H^{5}(\mathbb{R})$ by Assumption 5.1 and Lemmas 2.4 and 5.4. By Lemma 5.6(i) $B(\varphi(c))=y$ and it is clear that $\langle B v, v\rangle=0$ for $v \in V_{c, \varepsilon}$. Finally it follows from Lemma 5.6(iii) that $B$ commutes with $T$.

By the previous lemma we may define $R(\lambda, v)$ to be the solution of the equation

$$
\frac{d R}{d \lambda}=B(R)
$$


with initial data $R(0, v)=v \in V_{c, \varepsilon}$. It follows that

$$
\begin{aligned}
\text { (i) } & R \text { is } C^{1} \quad \text { for } \quad|\lambda|<\lambda_{0}(v) \text { for any } v \in V_{c, \varepsilon} \\
\text { (ii) } & R(\lambda, T(r) v)=T(r) R(\lambda, v) \text { for all } v \in V_{c, \varepsilon}, r \in \mathbb{R} \\
\text { (iii) } & Q(R(\lambda, v))=Q(v) \\
\text { (iv) } & \frac{\partial R}{\partial \lambda}(0, \varphi(c))=y .
\end{aligned}
$$

Lemma 5.8 If $d^{\prime \prime}(c)<0$ then there exist $\varepsilon>0$ and a $C^{1}$ functional $\lambda: V_{c, \varepsilon} \rightarrow \mathbb{R}$ such that for $v \in V_{c, \varepsilon}$

$$
K(R(\lambda(v), v))=K(\varphi(c))=\frac{2}{p-1} d(c)
$$

Proof. Since $R(0, \varphi(c))=\varphi(c)$ the lemma follows from the Implicit Function Theorem if we can show that

$$
\left.\frac{\partial K}{\partial \lambda}(R(\lambda, \varphi(c)))\right|_{\lambda=0} \neq 0
$$

By (5.4), the homogeneity of $K$ and (3.4) we have

$$
\begin{aligned}
\left.\frac{\partial K}{\partial \lambda}(R(\lambda, \varphi(c)))\right|_{\lambda=0} & =\left\langle K^{\prime}(\varphi(c)), y\right\rangle \\
& =h^{\prime}(0)\left\langle K^{\prime}(\varphi(c)), \varphi^{\prime}(c)\right\rangle+\left\langle K^{\prime}(\varphi(c)), \varphi(c)\right\rangle \\
& =h^{\prime}(0) \frac{d}{d c} K(\varphi(c))+(p+1) K(\varphi(c)) \\
& =\frac{2}{p-1}\left(h^{\prime}(0) d^{\prime}(c)+(p+1) d(c) .\right)
\end{aligned}
$$

Since $d(c)>0$ we only need to show that $h^{\prime}(0) d^{\prime}(c)>0$. Using (3.7), (5.4) and Lemma 5.5 (ii) we find that

$$
\begin{aligned}
h^{\prime}(0) d^{\prime}(c) & =h^{\prime}(0) Q(\varphi(c))=\frac{1}{2} h^{\prime}(0)\langle\varphi(c), \varphi(c)\rangle \\
& =\frac{1}{2} h^{\prime}(0)\left\langle\varphi(c),-h^{\prime}(0) \varphi^{\prime}(c)\right\rangle \\
& =-\frac{1}{2}\left(h^{\prime}(0)\right)^{2} d^{\prime \prime}(c)>0
\end{aligned}
$$

which proves the lemma.

Lemma 5.9 If $d^{\prime \prime}(c)<0$ then the functional $\lambda$ defined in Lemma 5.8 satisfies

$$
E(R(\lambda(v), v)) \geq E(\varphi(c))
$$

for $v \in V_{c, \varepsilon}$ with $Q(v)=Q(\varphi(c))$. 
Proof. By Lemma 5.8, $K(R(\lambda(v), v))=K(\varphi(c))$ and, since $I_{c}$ is minimized at $\varphi(c)$ subject to this constraint, we have

$$
\begin{aligned}
E(R(\lambda(v), v)) & =I_{c}(R(\lambda(v), v))-K(R(\lambda(v), v))-c Q(R(\lambda(v), v)) \\
& \geq I_{c}(\varphi(c))-c Q(\varphi(c))-K(\varphi(c)) \\
& =E(\varphi(c))
\end{aligned}
$$

and the lemma is proved.

Lemma 5.10 Let $v \in V_{c, \varepsilon}$ with $Q(v)=Q(\varphi(c))$ and $\lambda(v) \neq 0$. If $d^{\prime \prime}(c)<0$ then

$$
E(\varphi(c))<E(v)+\lambda(v) P(v)
$$

where $P(v)=\left\langle E^{\prime}(v), B(v)\right\rangle$ and $\lambda$ is defined in Lemma 5.8 .

Proof. This follows by computing a second order Taylor expansion of $E(R(\lambda, v))$ at $\lambda=0$. For any $v$ we have

$$
\left.\frac{\partial}{\partial \lambda} E(R(\lambda, v))\right|_{\lambda=0}=\left\langle E^{\prime}\left(R(0, v), \frac{\partial R}{\partial \lambda}(0, v)\right\rangle=\left\langle E^{\prime}(v), B(v)\right\rangle=P(v)\right.
$$

Also, since $Q(R(\lambda, \varphi(c)))=Q(\varphi(c))$ we have

$$
\frac{\partial}{\partial \lambda} Q(R(\lambda, \varphi(c)))=\frac{\partial^{2}}{\partial \lambda^{2}} Q(R(\lambda, \varphi(c)))=0
$$

for all $\lambda$. Since $R(0, \varphi(c))=\varphi(c)$ it follows as in the proof of Lemma 5.5(i), making use of the fact that $\varphi(c)$ solves (1.8), that

$$
\left.\frac{\partial^{2}}{\partial \lambda^{2}} E(R(\lambda, \varphi(c)))\right|_{\lambda=0}=\left\langle H_{c} y, y\right\rangle<0 .
$$

So, for $\lambda \neq 0$ small and $\varepsilon>0$ small it follows that if $v \in V_{c, \varepsilon}$ then

$$
E(R(\lambda, v))<E(v)+\lambda P(v) .
$$

If $v$ also satisfies $Q(v)=Q(\varphi(c))$ then Lemma 5.9 implies

$$
E(\varphi(c)) \leq E(R(\lambda(v), v))<E(v)+\lambda(v) P(v)
$$

and the lemma is proved.

Lemma 5.11 There curve $\psi(\sigma)$ defined by $(5.6)$ maps $(-\delta, \delta)$ to $H^{2}(\mathbb{R})$ and satisfies

$$
\begin{aligned}
(i) & \psi(0)=\varphi(c) \\
(\text { ii }) & E(\psi(\sigma))<E(\varphi(c)) \quad \text { for } \quad \sigma \neq 0 \\
(\text { iii }) & Q(\psi(\sigma))=Q(\varphi(c)) \\
(\text { iv }) & P(\psi(\sigma)) \text { changes sign at } \sigma=0
\end{aligned}
$$


Proof. It was shown in the proof of Lemma 5.5 that (i) and (iii) hold. Since $E(\psi(\sigma))=\mathcal{E}(\sigma)$ by (5.7), (ii) follows from (5.8) and Lemma 5.5(i). Since the function $\lambda(\psi(\sigma))$ is defined by

$$
K(\varphi(c))=K(R(\lambda(\psi(\sigma)), \psi(\sigma)))
$$

differentiating at $\sigma=0$ gives

$$
\begin{aligned}
0 & =\left\langle K^{\prime}(\varphi(c)),\left.\left(\frac{\partial R}{\partial \lambda} \frac{\partial \lambda(\psi(\sigma))}{\partial \sigma}+\frac{\partial R}{\partial v} \frac{\partial \psi(\sigma)}{\partial \sigma}\right)\right|_{\sigma=0}\right\rangle \\
& =\left(\left.\frac{\partial \lambda(\psi(\sigma))}{\partial \sigma}\right|_{\sigma=0}+1\right)\left\langle K^{\prime}(\varphi(c)), y\right\rangle
\end{aligned}
$$

But the last pairing was shown to be positive in the proof of Lemma 5.8. Thus

$$
\left.\frac{\partial \lambda(\psi(\sigma))}{\partial \sigma}\right|_{\sigma=0}=-1
$$

so that $\lambda(\psi(\sigma))$ changes sign at $\sigma=0$. This also shows that $\lambda(\psi(\sigma)) \neq 0$ for $\sigma \neq 0$ small. Thus, since $E(\psi(\sigma))$ is maximized at $\sigma=0$ Lemma 5.10 shows that

$$
\lambda(\psi(\sigma)) P(\psi(\sigma))>0 .
$$

and therefore $P(\psi(\sigma))$ also changes sign at $\sigma=0$.

We now establish a crucial decay estimate on solutions of (1.1) with special initial data. We first quote a lemma from [28] concerning the decay rate of solutions to the linear equation.

Lemma 5.12 Let $U_{0}(t)$ denote the evolution operator for the linear equation

$$
u_{t}+u_{x x x x x}+b u_{x x x}=0
$$

Then, for all $t>0, U_{0}(t)$ is bounded from $L^{1}(\mathbb{R})$ to $L^{\infty}(\mathbb{R})$ and

$$
\left\|U_{0}(t)\right\|_{1, \infty} \leq C(1+t)^{-\frac{1}{1+\mu}}
$$

where $\mu=2$ if $b \neq 0$ and $\mu=4$ if $b=0$.

It is convenient to write (1.1) as

$$
u_{t}-M u_{x}=\left(f\left(u, u_{x}, u_{x x}\right)\right)_{x}
$$

where $M=-\partial_{x}^{4}-b \partial_{x}^{2}$. The next lemma shows that solutions with special initial data obey a sublinear growth estimate. See Theorem 4.3 of [28].

Lemma 5.13 For $p \geq 2$ let $g \in H^{2}(\mathbb{R}) \cap L^{1}(\mathbb{R})$ and suppose $M g \in L^{1}(\mathbb{R})$. If $u(t)$ is the solution of (1.1) with initial data $u(0)=g$, then

$$
\sup _{-\infty<z<\infty}\left|\int_{-\infty}^{z} u(x, t) d x\right| \leq C\left(1+t^{\frac{\mu}{1+\mu}}\right)
$$

for $0 \leq t<T$ where $T$ is the existence time for $u, \mu$ is as in Lemma 5.12 and where $C$ is a constant depending only on $\sup _{0 \leq \tau \leq t}\|u(\tau)\|_{H^{2}(\mathbb{R})},\|g\|_{L^{1}(\mathbb{R})}$ and $\|M g\|_{L^{1}(\mathbb{R})}$. 
Proof. The solution $u$ satisfies

$$
\begin{aligned}
u(t) & =U_{0}(t) g+\int_{0}^{t} U_{0}(t-\tau)\left(f\left(u, u_{x}, u_{x x}\right)\right)_{x} d \tau \\
& =U_{0}(t) g+\partial_{x} \int_{0}^{t} U_{0}(t-\tau) f\left(u, u_{x}, u_{x x}\right) d \tau
\end{aligned}
$$

Define $U(x, t)=\int_{-\infty}^{x} u(\xi, t) d \xi$ and $Z(x, t)=\int_{-\infty}^{x}\left(U_{0}(t) g\right)(\xi) d \xi$. We then have

$$
U(t)=Z(x, t)+\int_{0}^{t} U_{0}(t-\tau) f\left(u, u_{x}, u_{x x}\right) d \tau
$$

Since $U_{0}(t) g$ solves the linear equation we have

$$
\begin{aligned}
Z(x, t) & =\int_{-\infty}^{x} g(\xi) d \xi+\int_{0}^{t}\left(M U_{0}(\tau) g\right)(x) d \tau \\
& =\int_{-\infty}^{x} g(\xi) d \xi+\int_{0}^{t}\left(U_{0}(\tau) M g\right)(x) d \tau
\end{aligned}
$$

and therefore by Lemma 5.12

$$
\begin{aligned}
|Z(x, t)| & \leq\|g\|_{L^{1}(\mathbb{R})}+C \int_{0}^{t}(1+\tau)^{-\frac{1}{1+\mu}}\|M g\|_{L^{1}(\mathbb{R})} d \tau \\
& \leq C(1+t)^{\frac{\mu}{1+\mu}}\left(\|g\|_{L^{1}(\mathbb{R})}+\|M g\|_{L^{1}(\mathbb{R})}\right)
\end{aligned}
$$

Using Lemma 5.12 again we see that

$$
\left|\int_{0}^{t} U_{0}(t-\tau) f\left(u, u_{x}, u_{x x}\right) d \tau\right| \leq C(1+t)^{\frac{\mu}{1+\mu}} \sup _{\tau \in[0, t]}\left\|f\left(u(\tau), u_{x}(\tau), u_{x x}(\tau)\right)\right\|_{L^{1}(\mathbb{R})}
$$

By (2.8) and the Sobolev inequality we have

$$
\left\|f\left(u(\tau), u_{x}(\tau), u_{x x}(\tau)\right)\right\|_{L^{1}(\mathbb{R})} \leq C\|u(\tau)\|_{H^{2}(\mathbb{R})}
$$

for $p \geq 2$.

Proof of Theorem 5.3. We proceed by contradiction. Suppose $\mathcal{T}_{c}$ is stable and choose initial data $g$ on the curve $\psi(\sigma)$ sufficiently close to $\varphi(c)$. Let $u(t)$ denote the solution of (1.1) with initial data $u(0)=g$, which we may assume (by stability) to exist on the interval $[0, \infty)$ and satisfy $u(t) \in V_{c, \varepsilon}$ for all $t$. By the definition of $\psi$ and by our assumptions on $\varphi(c)$ we have $\|g\|_{L^{1}(\mathbb{R})} \leq C$. Also, since $\psi(\sigma)$ is a linear combination of $\varphi(h(\sigma))$ and $\varphi(c),(1.5)$ implies

$M \psi(\sigma)=\sigma c \varphi(c)-\sigma f\left(\varphi(c), \varphi(c)_{x}, \varphi(c)_{x x}\right)+h(\sigma) \varphi(h(\sigma))-f\left(\varphi(h(\sigma)), \varphi(h(\sigma))_{x}, \varphi(h(\sigma))_{x x}\right)$

Hence, using (2.8) again, it follows that $\|M g\|_{L^{1}(\mathbb{R})}$ is bounded by the $L^{1}$ and $H^{2}$ norms of both $\varphi(c)$ and $\varphi(h(\sigma))$, which are bounded by Lemma 2.4. Next define

$$
Y(x)=\int_{-\infty}^{x} y(z) d z,
$$


where $y$ is the unstable direction constructed in Lemma 5.5 and let

$$
A(t)=\int_{-\infty}^{\infty} Y(x-s(u(t))) u(x, t) d x=\langle T(-s(u(t))) Y, u(t)\rangle .
$$

We now show (as in [7]) that $A(t)$ converges by letting $\gamma=\int_{-\infty}^{\infty} y(x) d x$ and writing

$$
A(t)=\int_{-\infty}^{\infty}(Y(x-s(u(t)))-\gamma \mathcal{H}(x-s(u(t)))) u(x, t) d x+\gamma \int_{s(u(t))}^{\infty} u(x, t) d x
$$

where $\mathcal{H}$ denotes the Heaviside function. By the assumption of stability we have that $\|u(t)\|_{H^{2}(\mathbb{R})} \leq C$ for all $t$ and thus Lemma 5.13 implies that

$$
|A(t)| \leq\|Y-\gamma \mathcal{H}\|_{L^{2}(\mathbb{R})}\|u(t)\|_{L^{2}(\mathbb{R})}+C\left(1+t^{\frac{\mu}{1+\mu}}\right)
$$

To show that $Y-\gamma \mathcal{H} \in L^{2}(\mathbb{R})$ notice that

$$
\begin{aligned}
\|Y-\gamma \mathcal{H}\|_{L^{2}(\mathbb{R})} & =\left(\int_{-\infty}^{0}\left(\int_{-\infty}^{x} y(z) d z\right)^{2} d x+\int_{0}^{\infty}\left(\int_{x}^{\infty} y(z) d z\right)^{2} d x\right)^{1 / 2} \\
& =\left(\int_{-\infty}^{\infty}\left(\int_{-\infty}^{\infty} k(x, z) d z\right)^{2} d x\right)^{1 / 2}
\end{aligned}
$$

where $k(x, z)=y(z) \chi\{z / x>1\}$. By Minkowski's inequality the last term is bounded by

$$
\begin{aligned}
\int_{-\infty}^{\infty}\left(\int_{-\infty}^{\infty}(k(x, z))^{2} d x\right)^{1 / 2} d z & =\int_{-\infty}^{0}\left(\int_{z}^{0}(y(z))^{2} d x\right)^{1 / 2} d z+\int_{0}^{\infty}\left(\int_{0}^{z}(y(z))^{2} d x\right)^{1 / 2} d z \\
& =\int_{-\infty}^{\infty}|z|^{1 / 2}|y(z)| d z
\end{aligned}
$$

which is bounded by Lemma 5.5, Lemma 2.4, Assumption 5.1 and Lemma 5.4. Hence we have

$$
|A(t)| \leq C\left(1+t^{\frac{\mu}{1+\mu}}\right)
$$

for $0 \leq t<\infty$. Differentiating (5.11) in $t$ and using (5.9), we see that

$$
\begin{aligned}
\frac{d A}{d t} & =\left\langle T(-s(u(t))) Y, u_{t}(t)\right\rangle-\langle T(-s(u(t))) y, u(t)\rangle\left\langle s^{\prime}(u(t)), u_{t}(t)\right\rangle \\
& =\left\langle T(-s(u(t))) Y-\langle T(-s(u(t))) y, u(t)\rangle s^{\prime}(u(t)), J E^{\prime}(u(t))\right\rangle \\
& =\left\langle T(-s(u(t))) y-\langle T(-s(u(t))) y, u(t)\rangle \partial_{x} s^{\prime}(u(t)), E^{\prime}(u(t))\right\rangle \\
& =\left\langle B(u(t)), E^{\prime}(u(t))\right\rangle=P(u(t))
\end{aligned}
$$

By Lemma 5.11(ii) and the invariance of $E$, we have

$$
0<C_{0}=E(\varphi(c))-E(g)=E(\varphi(c))-E(u(t))
$$

for some constant $C_{0}$ and by the invariance of $Q$ we may apply Lemma 5.10 to conclude that

$$
\lambda(u(t)) P(u(t))>C_{0} \quad \text { for } \quad t \in[0, \infty) .
$$


Thus both $\lambda(u(t))$ and $P(u(t)$ do not change sign. Without loss of generality assume that both are positive. Then by choosing $\varepsilon$ smaller if necessary we may assume that $0<\lambda(u(t))<$ 1. So, for $u(t) \in V_{c, \varepsilon}$, we have

$$
\frac{d A}{d t} \geq C_{0}>0
$$

Together with (5.12) this implies that $u(t)$ leaves $V_{c, \varepsilon}$ in finite time, a contradiction to the stability assumption. Hence $\mathcal{T}_{c}$ must be unstable.

Corollary 5.14 Suppose $b=0$ and $F(q, r)$ satisfies (1.3), (2.2) and is homogeneous in $q$ of degree $\alpha$ and homogeneous in $r$ of degree $\beta$, where $\alpha+\beta=p+1>2$. Then solitary waves of any speed $c>0$ are stable if $\alpha+3 \beta<10$ and unstable if $\alpha+3 \beta>10$.

In particular, if $F(q, r)$ is a homogeneous polynomial, we have stability only in the cases

$$
\begin{array}{cc}
u^{\alpha} & \text { for } 3 \leq \alpha \leq 9 \\
u^{\alpha} u_{x} & \text { for } 2 \leq \alpha \leq 6 \\
u^{\alpha} u_{x}^{2} & \text { for } 1 \leq \alpha \leq 3 \\
u_{x}^{3} &
\end{array}
$$

and instability otherwise. Thus the equations studied by Olver, Zufiria and Hunter \& Scheurle admit stable solitary waves.

Proof. By Lemma $3.3 d(c)$ is convex when $\gamma>1$, which occurs when $\alpha+3 \beta<10$.

The regions of stability $\mathcal{S}$ and instability $\mathcal{I}$ are shown in Figure 1.

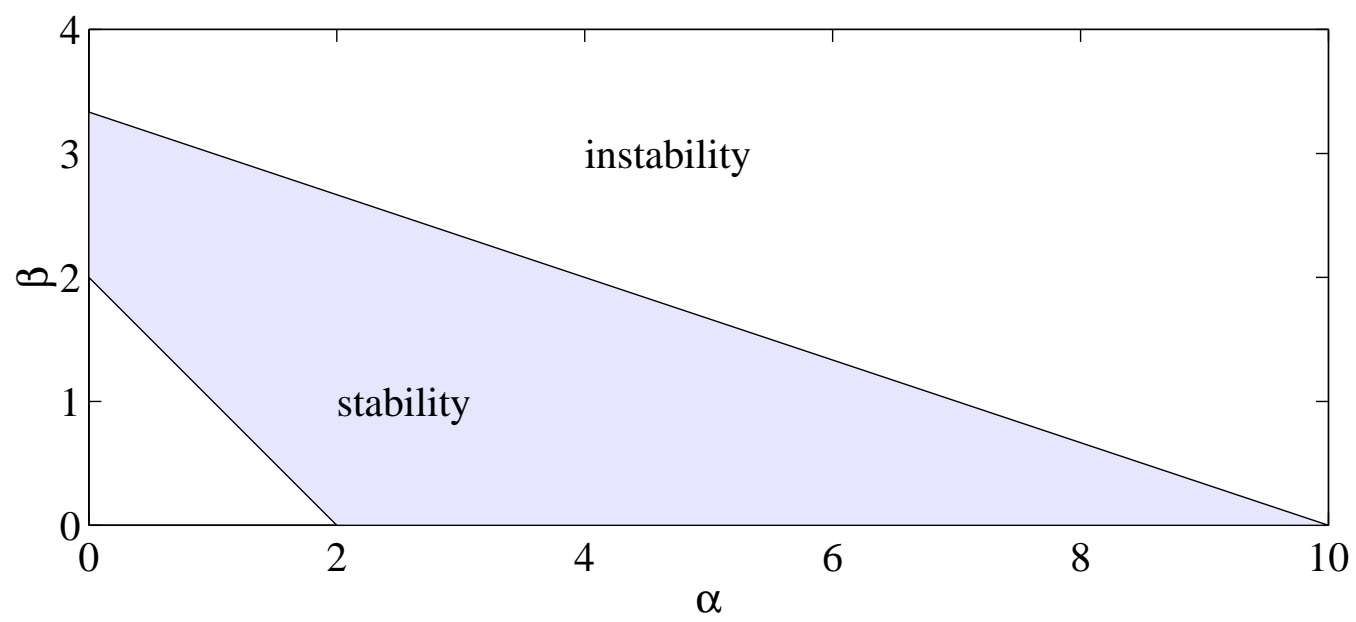

Figure 1: Regions of stability and instability.

\section{References}

[1] J.P. Albert, J.L. Bona And D.B. Henry (1987). Sufficient conditions for stability of solitary-wave solutions of model equations for long waves. Physica $D$ 24, no. 1-3, 343-366 
[2] C.J. Amick And K. Kirchgässner (1989). A theory of solitary water-waves in the presence of surface tension. Arch. Rat. Mech. Anal. 105, no. 1, 1-49

[3] C.J. Amick and J.F. Toland (1992). Homoclinic orbits in the dynamic phase-space analogy of an elastic strut. European J. Appl. Math. 3, no. 2, 97-114

[4] T.B. Benjamin (1972). The stability of solitary waves. Proc. Roy. Soc. Lond. Ser. A 328 153-183

[5] D.J. Benney (1977). A general theory for interactions between short and long waves. Stud. Appl. Math. 56, 81-94

[6] J. Bona (1975). On the stability of solitary waves. Proc. Roy. Soc. Lond. Ser. A 344 363-374

[7] J.L. Bona P.E. Souganidis And W.A. Strauss (1987). Stability and instability of solitary waves of Korteweg-de Vries type Proc. Roy. Soc. London A 411 no. 1841, 395-412

[8] B. Buffoni, M.D. Groves and J.F. Toland (1996). A plethora of solitary gravitycapillary water waves with nearly critical bond and froude numbers. Phil. Trans. Roy. Soc. Lond. Ser. A 354, 575-607

[9] T. Cazenave And P.L. Lions (1982). Orbital stability of standing waves for some nonlinear Schrodinger equations Commun. Math. Phys. 85, 549-561

[10] A.R. Champneys and M.D. Groves (1996). A global investigation of solitary-wave solutions to a two-parameter model for water waves. J. Fluid Mech. 342, 199-229

[11] W. Craig And M.D. Groves (1994). Hamiltonian long-wave approximations to the water-wave problem. Wave Motion 19, 367-389

[12] M. Grillakis J. Shatah and W. Strauss (1987). Stability theory of solitary waves in the presence of symmetry I. J. Funct. Anal. 74 no. 1, 160-197

[13] J. Hunter And J. Scheurle (1988). Existence of perturbed solitary wave solutions to a model equation for water waves. Physica D 32, 253-268

[14] T. Kato (1975). Quasi-linear equations of evolution, with applications to partial differential equations, Lecture Notes in Math., Vol 448, Springer, Berlin

[15] T. Kato (1979). On the Korteweg-DeVries equation. Manuscripta Math. 28 no. 1-3, 89-99

[16] S. Kichenassamy (1997). Existence of solitary waves for water-wave models. Nonlinearity 10, no. 1, 133-151

[17] S. Kichenassamy and P. Olver (1992). Existence and nonexistence of solitary wave solutions to higher-order model evolution equations SIAM J. Math. Anal. 23, 1141-1166 
[18] P.D. LAx (1968). Integrals of nonlinear equations of evolution and solitary waves. Comm. Pure Appl. Math. 21, 467-490

[19] S.P. Levandosky (1998). Stability and instability of fourth-order solitary waves. J. Dynam. Diff. Eq. 10, no. 1, 151-188

[20] P.L. Lions (1984). The concentration-compactness principle in the calculus of variations. The locally compact case. Part I and Part II. Ann. Inst. Henri Poincare' Sect A (N.S.) 1, 109-145, 223-283

[21] Yue LIU (1997). Nonlinear stability of solitary waves of a generalized KadomtsevPetviashvili equation. Comm. Math. Phys. 183, 253-266

[22] P.J. McKenna And W. Walter (1990). Traveling waves in a suspension bridge SIAM J. Appl. Math. $50,703-715$

[23] P.J. Olver (1984). Hamiltonian and non-Hamiltonian models for water waves. Lecture Notes in Physics. No. 195, Springer Verlag. 273-290

[24] G. Ponce (1993). Lax pairs and higher order models for water waves. J. Diff. Eq. 102, no. $2,360-381$

[25] B. Sandstede (1997). Instability of localised buckling modes in a one-dimensional strut model. Phil. Trans. Roy. Soc. Lond. Ser. A 355 no. 1732, 2083-2097

[26] J. Shatah (1983). Stable standing waves of nonlinear Klein-Gordon equations. Comm. Math. Phys. 91 no. 3, 313-327

[27] J. Shatah and W. Strauss (1985). Instability of nonlinear bound states. Commun. Math. Phys. 100 no.2 , 173-190

[28] P.E. Souganidis And W. Strauss (1990). Instability of a class of dispersive solitary waves Proc. Roy. Soc. Edinburgh 114 A , 195-212

[29] M. Weinstein (1986). Lyapunov stability of ground states of nonlinear dispersive evolution equations. Comm. Pure Appl. Math. 39, 51-68

[30] M. Weinstein (1987). Existence and dynamic stability of solitary wave solutions of equations in long wave propagation. Comm. in P.D.E. 12, 1133-1173

[31] J.A. Zufiria (1987). Symmetry breaking in periodic and solitary gravity-capillary waves on water of finite depth. J. Fluid Mech 184, 183-206 